\title{
A scaffold for signaling of Tim-4-mediated efferocytosis is formed by fibronectin
}

\author{
Juyeon Lee ${ }^{1,2} \cdot$ Boyeon Park ${ }^{1,3} \cdot$ Byeongjin Moon ${ }^{1} \cdot$ Jeongjun Park ${ }^{1}$ Hyunji Moon ${ }^{1,2} \cdot$ Kwanhyeong Kim $^{1,2} \cdot$ \\ Sang-Ah Lee ${ }^{1} \cdot$ Deokhwan Kim $^{1,2} \cdot$ Chanhyuk Min ${ }^{1} \cdot$ Dae-Hee Lee $^{4} \cdot$ Gwangrog Lee $^{1} \cdot$ Daeho Park $^{1,2}$
}

Received: 9 August 2018 / Revised: 21 October 2018 / Accepted: 29 October 2018 / Published online: 19 November 2018

(c) ADMC Associazione Differenziamento e Morte Cellulare 2018

\begin{abstract}
An essential step during clearance of apoptotic cells is the recognition of phosphatidylserine (PS) exposed on apoptotic cells by its receptors on phagocytes. Tim- 4 directly binding to PS and functioning as a tethering receptor for phagocytosis of apoptotic cells has been extensively studied over the past decade. However, the molecular mechanisms by which Tim- 4 collaborates with other engulfment receptors during efferocytosis remain elusive. By comparing efferocytosis induced by Tim-4 with that by Anxa5-GPI, an artificial tethering receptor, we found that Tim-4 possesses auxiliary machinery to induce a higher level of efferocytosis than Anxa5-GPI. To search for that, we performed a yeast two-hybrid screen and identified Fibronectin (Fn1) as a novel Tim-4-associating protein. Tim-4 directly associated with Fn1 and formed a complex with integrins via the association of Fn1. Through Tim- $4^{-1-}$ mice and cell-based assays, we found that modulation of the Fn1 level affected efferocytosis induced by Tim- 4 and disruption of the interaction between Tim- 4 and Fn1 abrogated Tim-4mediated efferocytosis. In addition, Tim- 4 depletion attenuated integrin signaling activation and perturbation of integrin signaling suppressed Tim-4-promoted efferocytosis. Taken together, the data suggest that Fn1 locates Tim- 4 and integrins in close proximity by acting as a scaffold, resulting in synergistic cooperation of Tim-4 with integrins for efficient efferocytosis.
\end{abstract}

These authors contributed equally: Juyeon Lee, Boyeon Park

Edited by E. Baehrecke

Electronic supplementary material The online version of this article (https://doi.org/10.1038/s41418-018-0238-9) contains supplementary material, which is available to authorized users.

Daeho Park

daehopark@gist.ac.kr

1 School of Life Sciences and Aging Research Institute, Gwangju Institute of Science and Technology, Gwangju 61005, Korea

2 Research Center for Cellular Homeostasis, Ewha Womans University, Seoul 03760, Korea

3 Microbiology and Functionality Research Group, World Institute of Kimchi, Gwangju 61755, Korea

4 Department of Oncology, College of Medicine, Korea University, Seoul 08308, Korea

\section{Introduction}

Apoptotic cells are efficiently cleared from the body by well-coordinated sequential processes called efferocytosis. An essential step in efferocytosis is the recognition of apoptotic cells by phagocytes [1-3]. In general, phosphatidylserine (PS), a best-characterized ligand on apoptotic cells, exposed on the outer leaflet of the plasma membrane of apoptotic cells interacts with PS receptors on phagocytes for their recognition by phagocytes [4-6]. Some of them especially called PS receptors, such as Tim-4, BAI1, and Stabilin-4, directly bind to PS [7-11]. Others, including TAM receptors, $\alpha_{v} \beta_{3}$, and $\alpha_{v} \beta_{5}$, indirectly associate with PS via bridging molecules, such as Gas6 and MFG-E8, which link PS on apoptotic cells to engulfment receptors on phagocytes [12-17].

Engulfment receptors for apoptotic cells could be classified into two groups based on their ability to directly transduce signals into phagocytes. Receptors in one group can directly transduce signals into phagocytes after binding to apoptotic cells. By contrast, receptors, called tethering receptors, in the other group 


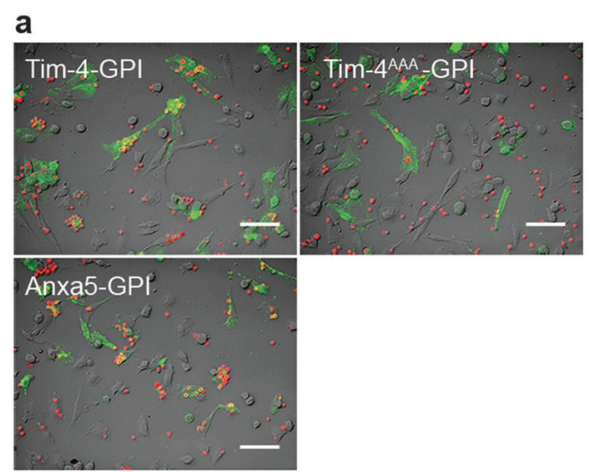

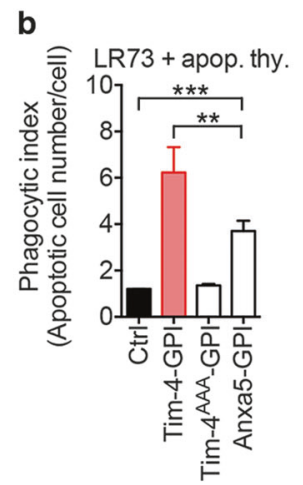

\section{C}

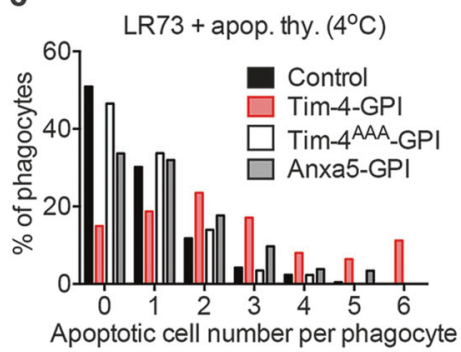

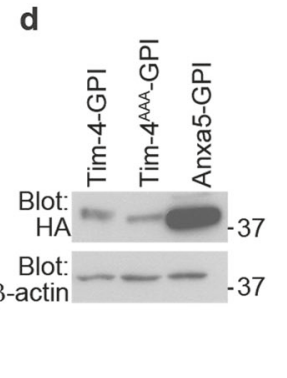

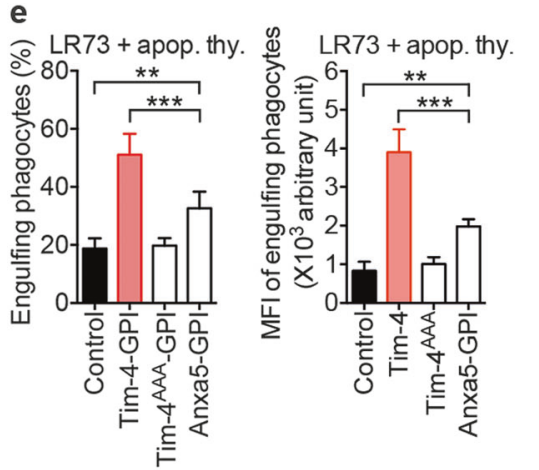

Fig. 1 Tim- 4 mediates efferocytosis more efficiently than Anxa5-GPI. a LR73 cells were transfected with the indicated plasmids, incubated with TAMRA-stained apoptotic thymocytes at $37^{\circ} \mathrm{C}$ for $2 \mathrm{~h}$, stained with an anti-HA antibody, and labeled with an Alexa Fluor 488conjugated secondary antibody. Scale bar, $50 \mu \mathrm{m}$. b Quantification of a. The number of apoptotic cells per phagocyte was counted for at least $100 \mathrm{HA}$-positive and -negative phagocytes in randomly selected areas. HA-negative cells were considered as control cells. $n=3$. c LR73 cells transfected with the indicated plasmids were incubated with TAMRA-stained apoptotic cells at $4{ }^{\circ} \mathrm{C}$ for $2 \mathrm{~h}$. The number of apoptotic cells binding to phagocytes were counted. d LR73 cells were transfected with the indicated plasmids and exogenous proteins were detected by immunoblotting. $\beta$-actin was used as a loading control. e LR73 cells transfected with the indicated plasmids were incubated with TAMRA-stained apoptotic thymocytes for $2 \mathrm{~h}$, stained with an Alexa Fluor 488-conjugated HA antibody, and analyzed by flow

bind to apoptotic cells but cannot directly transduce signals. They are thought to secure apoptotic cells to phagocytes, which facilitates recognition and ingestion of the apoptotic cells by other engulfment receptors $[8$, 18-22].

One of the most notable tethering receptors is T-cell immunoglobulin and mucin domain-containing 4 (Tim-4). Tim-4 is a transmembrane protein comprising a relative short cytoplasmic tail that lacks a signaling motif and an extracellular domain (ECD) containing an $\mathrm{IgV}$ domain and a mucin domain [23]. It was reported that Tim-4 functions as a PS receptor by binding PS on apoptotic cells $[8,24,25]$. Tim4 is considered to be a tethering receptor because engulfment of apoptotic cells mediated by truncated mutants of Tim- 4 lacking the cytoplasmic tail or transmembrane domain is cytometry. HA- and TAMRA-positive cells were considered to be phagocytes engulfing apoptotic cells (left). The MFI of apoptotic cells engulfed by phagocytes was evaluated (right). $n=5$. f PS was immobilized on a planar hydrophobic sensor chip. The purified ECD of Tim-4 or Anxa5 was flowed over the surface of the chip. Binding was monitored and analyzed using a surface plasmon resonance instrument (SR7500 DC, Reichert Inc.). g PS vesicles were added to LR73 cells transfected with the indicated plasmids, and then the cells were incubated for $2 \mathrm{~h}$, stained with an anti-HA antibody, and analyzed by flow cytometry. Cells positive for both HA staining and PS vesicles were considered to be phagocytes engulfing PS vesicles (left). The MFI of PS vesicles engulfed by phagocytes was measured (right). $n=3$. All data are shown as the mean \pm standard deviation and images are representative of at least three independent experiments. ${ }^{*} P<0.05$, $* * P<0.01, * * * P<0.001$

unimpaired [26]. Thus, the general consensus about signal transduction during Tim-4-induced efferocytosis is that Tim-4 tethers apoptotic cells to phagocytes and other engulfment receptors capable of signaling, such as integrins, thereafter efficiently recognize apoptotic cells and mediate their ingestion [20, 21]. A recent study strengthens this notion. It was reported that a co-receptor directly associated with a tethering receptor is dispensable and that a tethering receptor alone can promote engulfment of apoptotic cells by effectively securing these cells to phagocytes [19]. Nevertheless, it is uncertain if and how Tim- 4 collaborates with other engulfment receptors that can directly transduce signals.

In this study, efferocytosis induced by Tim- 4 was compared with that induced by Anxa5-GPI to determine whether Tim-4 has unappreciated machinery that promotes engulfment 
a

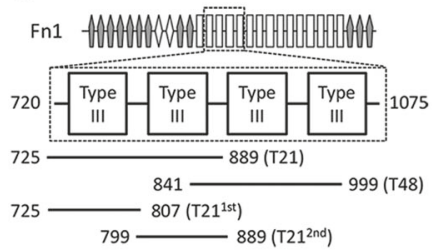

b

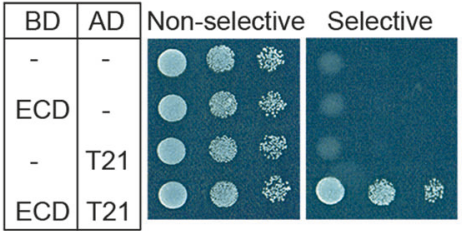

C

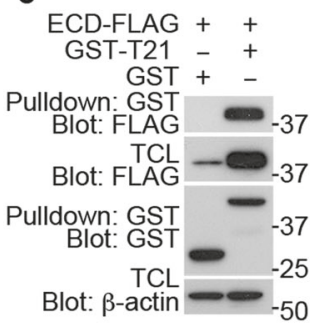

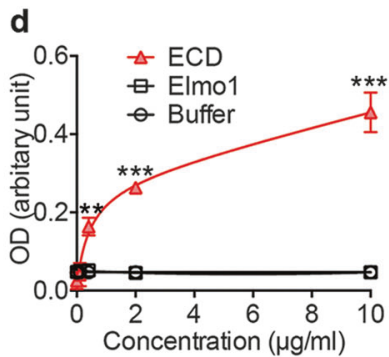

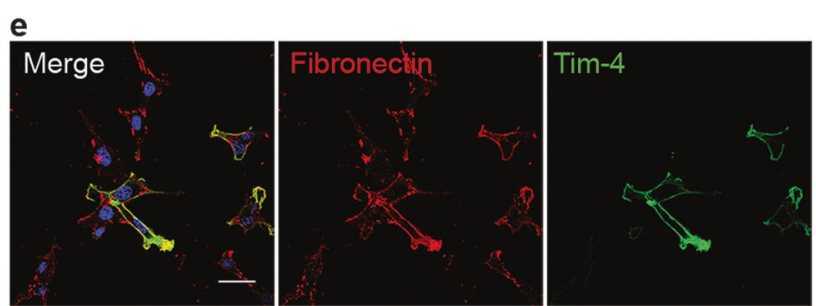

g

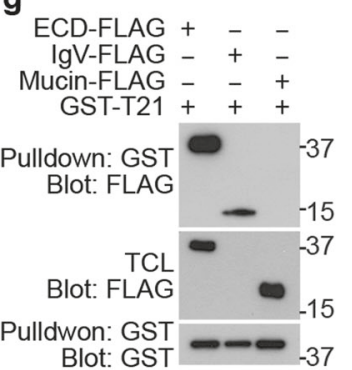

h

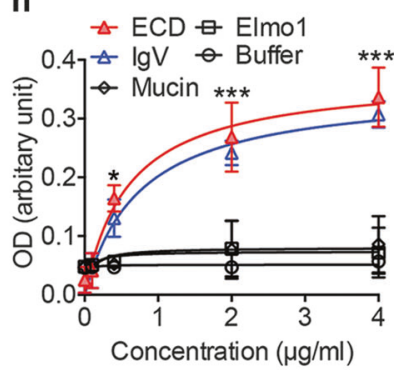

Fig. 2 The IgV domain of Tim- 4 associates with the type III domains of Fn1. a Schematic diagram showing the clones (T21 and T48) identified by the yeast two-hybrid screen. $\mathbf{b}$ Yeast transformed with the indicated plasmids was dotted on a selective plate containing $5 \mathrm{mM} 3-$ AT and lacking histidine, leucine, and tryptophan or on a non-selective plate lacking leucine and tryptophan. ECD, the ECD of Tim-4. c 293 $\mathrm{T}$ cells were transfected with the indicated plasmids and lysed 2 days later. Proteins in the cell lysates were precipitated with glutathionesepharose beads, and bound proteins were detected by immunoblotting. ECD, the ECD of Tim-4; TCL, total cell lysate. d Elmo1 or the purified ECD of Tim-4 was added to a Fn1-coated 96-well plate. Thereafter, the plate was incubated with an anti-FLAG antibody followed by a HRP-conjugated anti-mouse secondary antibody. The optical density of bound proteins at $450 \mathrm{~nm}$ was measured using a microplate reader. Elmo1 was used as a negative control. $n=3$. e NIH/ $3 \mathrm{~T} 3$ cells transfected with HA-Tim- 4 were incubated with anti-HA and anti-Fn1 antibodies, and then stained with Alexa Fluor 488- and Alexa

of apoptotic cells. Tim-4 induced a higher level of efferocytosis than Anxa5-GPI. By performing a yeast two-hybrid screen, we identified fibronectin (Fn1) as a Tim-4-interacting protein. Furthermore, we found that Tim-4 formed a complex with integrins through the Fn1 association. Using multiple in vivo and in vitro approaches, we propose that Fn1 functioning as a scaffold for Tim-4 and integrins promotes synergistic cooperation of these two types of engulfment receptors, leading to efficient binding and ingestion of apoptotic cells and eventually the marked level of Tim-4-mediated efferocytosis.
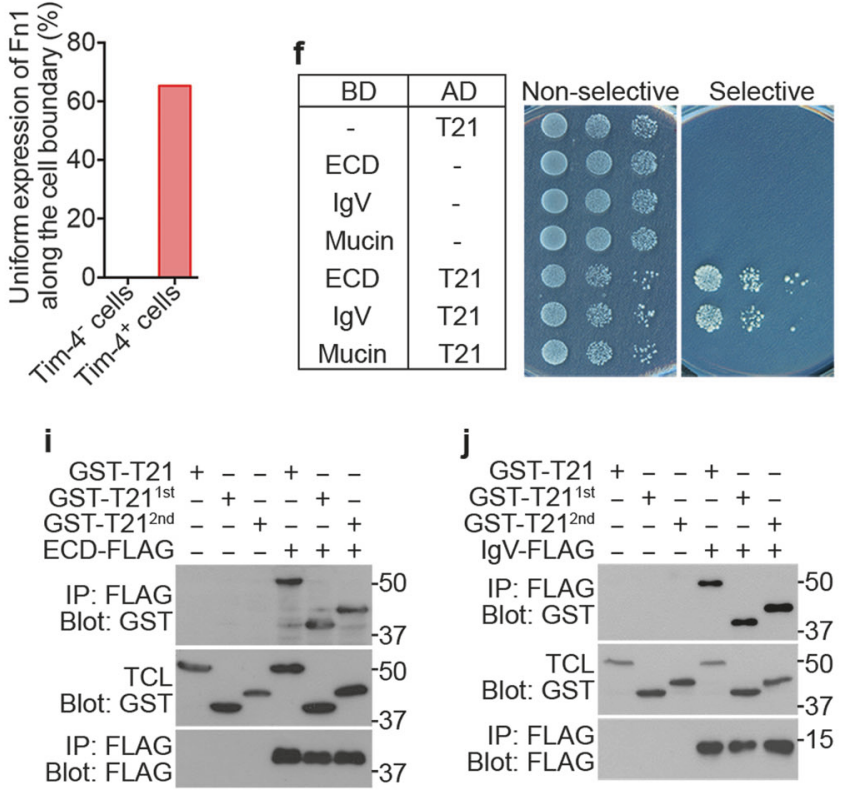

Fluor 594-conjugated secondary antibodies. Images were acquired by confocal microscopy (left). The ratio of cells uniformly expressing Fn1 along the cell boundary was calculated for at least 50 Tim-4-negative and -positive cells (right). Scale bar, $10 \mu \mathrm{m}$. f Yeast transformed with the indicated plasmids was dotted on a selective or non-selective plate. ECD, the ECD of Tim-4. g Lysates of 293 T cells transfected with the indicated plasmids were incubated with glutathione-sepharose beads. Bound proteins were detected by immunoblotting. $\mathbf{h}$ The indicated purified proteins were added to a Fn1-coated 96-well plate. The optical density of bound proteins at $450 \mathrm{~nm}$ was measured using a microplate reader. $\mathbf{i}$, j Lysates of $293 \mathrm{~T}$ cells transfected with the indicated plasmids were incubated with FLAG-conjugated agarose beads. Bound proteins were detected by immunoblotting. ECD, the ECD of Tim-4; TCL, total cell lysate; IP, immunoprecipitation. Data are shown as the mean \pm standard deviation and images are representative of at least three independent experiments. $* P<0.05, * * P<0.01, * * * P<0.001$

\section{Results}

\section{Tim-4 induces more-efficient efferocytosis than Anxa5-GPI}

It is unclear whether Tim-4 merely secures apoptotic cells to phagocytes or has unappreciated machinery to further promote engulfment of apoptotic cells. To investigate this, efferocytosis mediated by Tim-4-GPI, lacking the cytosolic tail and transmembrane domain, was compared with that mediated by Anxa5-GPI, an artificial tethering receptor 
sufficient to promote engulfment of apoptotic cells (Fig. S1a and S1b) [19]. LR73 cells expressing Tim-4-GPI, Tim$4^{\mathrm{AAA}}$-GPI (a PS-binding mutant), or Anxa5-GPI were incubated with apoptotic thymocytes and efferocytosis was evaluated. As expected, Tim-4-GPI-expressing cells ingested more apoptotic cells than Tim- ${ }^{\mathrm{AAA}}$-GPI-expressing or control cells (Fig. 1a, b). Interestingly, Tim-4-GPIexpressing cells phagocytosed more apoptotic cells than Anxa5-GPI-expressing cells. Although Anxa5-GPI also facilitated engulfment of apoptotic cells, its effect was inferior to that of Tim-4-GPI (Fig. 1a, b). In order to test whether the marked ingestion of apoptotic cells induced by Tim-4 results from increased binding of apoptotic cells to phagocyte, the cells were incubated with apoptotic cell at 4 ${ }^{\circ} \mathrm{C}$. About $25 \%$ of Tim-4-expressing cells had more than three apoptotic cells, whereas most of control or Anxa5GPI-expressing cells only had one or two apoptotic cells (Fig. 1c and S2), which indicates that Tim-4-GPI promotes binding of apoptotic cells more effectively than Anxa5-GPI, which leads to the marked level of efferocytosis.

These disparities in efferocytosis mediated by Tim-4-GPI versus Anxa5-GPI could be due to variation in their protein expression levels. Therefore, we investigated the expression levels of these receptors. Although their expression levels differed, that of Anxa5-GPI was always much higher than that of Tim-4-GPI (Fig. 1d). Moreover, when flow cytometry was used to gate cells with the same surface expression levels of Tim-4-GPI or Anxa5-GPI, the mean fluorescence intensity, indicative of the relative number of apoptotic cells per phagocyte, was higher for Tim-4-GPIexpressing cells than for Anxa5-GPI-expressing cells (Fig. 1e right), and a higher percentage of Tim-4-GPIexpressing cells than Anxa5-GPI-expressing cells ingested apoptotic cells (Fig. 1e left). This indicates that these disparities in efferocytosis are not owing to variation in protein expression levels.

The differences in the abilities of Tim-4-GPI and Anxa5GPI to induce efferocytosis may alternatively be explained by differences in their affinities for PS on apoptotic cells. Specifically, Tim-4 may have a stronger affinity for PS than Anxa5. To investigate this possibility, we compared the affinities of Tim-4 and Anxa5 for PS by performing surface plasmon resonance. Unexpectedly, the equilibrium dissociation constant $\left(\mathrm{K}_{\mathrm{D}}\right)$ of the ECD of Tim- 4 was $\sim 33$-fold higher than that of Anxa5 (Fig. 1f). This suggests that Tim4 has a lower affinity for PS than Anxa5, meaning that the marked efferocytosis induced by Tim-4-GPI is unlikely because it has an enhanced affinity for PS.

Next, a property of apoptotic cells rather than PS exposed on them might underlie the marked level of Tim-4GPI-mediated efferocytosis. To investigate this possibility, we generated PS vesicles containing PS and phosphatidylcholine (PC) to simplify complicated apoptotic cell contexts in efferocytosis. A higher percentage of Tim-4GPI-expressing cells than Anxa5-GPI-expressing cells engulfed PS vesicles (Fig. 1g left), and the number of PS vesicles ingested per cell was higher for the former cells than for the latter cells (Fig. 1g right). By contrast, engulfment of PC vesicles was negligibly induced by Tim4-GPI or Anxa5-GPI (Fig. S3). These data imply that PS, and no other factor, on apoptotic cells is necessary and sufficient for the marked level of Tim-4-GPI-mediated efferocytosis. Taken together, these data suggest that efficient and effective efferocytosis is not determined just by the PS affinities or the expression levels of tethering receptors and that the ECD of Tim- 4 could possess an unidentified component.

\section{Tim-4 associates with Fn1}

To search for a component interacting with the ECD of Tim-4, we performed a yeast two-hybrid screen using the ECD of Tim- 4 as bait. Two candidate clones, T21 and T48, identified by the screen contained amino acids $725-889$ and 841-999 of Fn1, respectively (Fig. 2a). Because T21 contained two intact fibronectin type III domains, we used T21 for further experiments. Yeast expressing both the ECD of Tim-4 and T21 grew on selective plates, whereas that expressing only the ECD of Tim-4 or T21 did not (Fig. 2b), which indicate that the ECD of Tim-4 interacts with this fragment of Fn1.

Next, we examined whether this interaction occurs in mammalian cells. FLAG-tagged ECD expressed in 293T HEK cells was co-precipitated with GST-tagged T21, but not with GST (Fig. 2c), indicating that these proteins also associate in mammalian cells. The expression level of FLAG-tagged ECD was high in the presence of GST-T21. This might be because this interaction increases the stability of the ECD protein. We further tested whether the ECD of Tim-4 directly associates with full-length Fn1 by performing an enzyme-linked immunosorbent assay. Absorbance increased in a dose-dependent manner by adding the purified ECD of Tim- 4 to a plate coated with soluble fulllength Fn1. By contrast, absorbance generated upon addition of Elmo1 or upon incubating the ECD of Tim-4 with laminin was indistinguishable from that generated upon addition of the buffer control (Fig. 2d and S4). Furthermore, we tested whether the proteins associate in cells. Endogenous Fn1 and exogenous Tim- 4 colocalized along the boundary of NIH/3T3 cells (Fig. 2e left and S5). Intriguingly, Fn1 localized uniformly along the cell boundary in > $65 \%$ of cells exogenously expressing Tim- 4 , but nonuniformly in the absence of Tim-4 (Fig. 2e right). In addition, we also harnessed the proximity ligation assay to further test the interaction of Tim-4 with Fn1. Fluorescence generated by Tim- 4 and Fn1 was as strong as that generated 

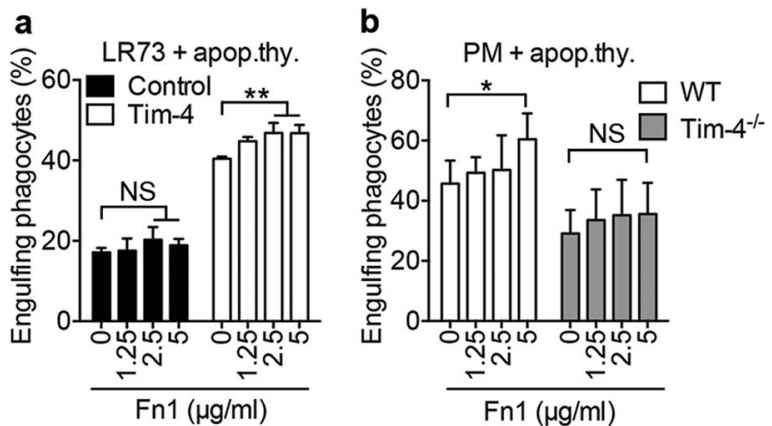

Fig. 3 The level of Fn1 influences Tim-4-mediated efferocytosis. a, b LR73 cells transfected with Tim- 4 a or peritoneal macrophages derived from WT or Tim- $4^{-/-}$mice $\mathbf{b}$ were incubated with TAMRAstained apoptotic thymocytes and the indicated concentrations of Fn1 for $2 \mathrm{~h}(\mathbf{a}, n=3)$ or $15 \mathrm{~min}(\mathbf{b}, n=3)$, stained with an anti-HA antibody a, and analyzed by flow cytometry. $\mathbf{c}$ Total RNA was prepared from L cells nucleofected with control or Fnl-targeting siRNA and cultured for $2 \mathrm{~d}$. The transcript levels of Fn1 were measured by

by integrins and Fn1 used as a positive control (Fig. S6). Collectively, these data suggest that Tim-4 directly associates with Fn1.

\section{The IgV domain of Tim-4 interacts with the type III domains of Fn1}

Next, we narrowed which of the domains of the ECD of Tim-4 is necessary for the interaction with Fn1. Yeast expressing $\mathrm{T} 21$ and the ECD or the $\operatorname{IgV}$ domain grew on selective plates, whereas yeast expressing T21 and the mucin domain did not (Fig. 2f), indicating that the $\operatorname{IgV}$ domain of Tim-4 interacts with this fragment of Fn1. The interaction between the $\operatorname{IgV}$ domain and $\mathrm{T} 21$ also observed in mammalian cells. Although the $\operatorname{IgV}$ domain was undetectable in cell lysates and was only observed by immunoprecipitation (Fig. S7), it was readily detected in precipitates of GST-T21 (Fig. 2g). The direct interaction between the $\operatorname{IgV}$ domain and Fn1 was further confirmed by enzyme-linked immunosorbent assay. Increased absorbance was observed only when the IgV domain and the ECD of Tim- 4 was incubated, but the mucin domain failed to increase absorbance (Fig. 2h). These data suggest that there is strong and direct interaction between the $\mathrm{IgV}$ domain of Tim- 4 and Fn1.

Next, we split T21 into two fragments, each containing one fibronectin type III domain, to identify which region is important for the interaction. Both fragments were coprecipitated with the ECD or the IgV domain of Tim-4 (Fig. 2i, j). Overall, these data indicate that the association of Tim- 4 with Fn1 is mediated by an interaction between the IgV domain of Tim- 4 and the type III domains of Fn1.
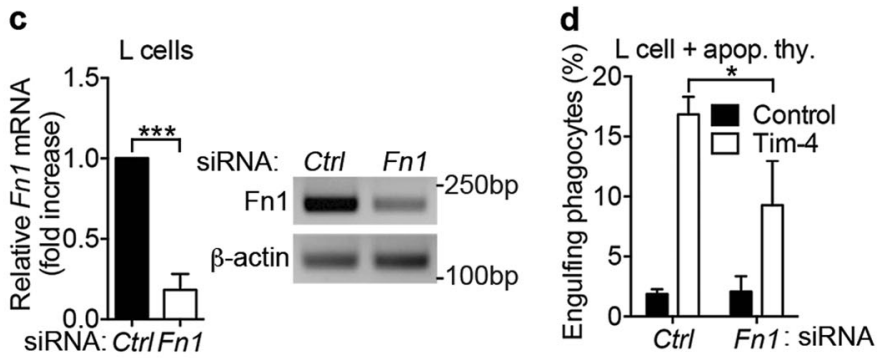

quantitative real-time polymerase chain reaction (left) and agarose-gel electrophoresis after polymerase chain reaction (right). Ctrl, control. $n$ $=3$. $\mathbf{d} \mathrm{L}$ cells nucleofected with Tim-4 and control or Fn1-targeting siRNA were incubated with apoptotic thymocytes, stained with an anti-HA antibody, and analyzed by flow cytometry. $n=3$. Data are shown as the mean \pm standard deviation and images are representative of at least three independent experiments. $* P<0.05$, $* * P<0.01$, *** $P$ $<0.001$

\section{The level of Fn1 influences Tim-4-mediated efferocytosis}

Next, we investigated the effects of Fn1 on Tim-4mediated efferocytosis. Addition of Fn1 further promoted efferocytosis enhanced by Tim-4 in a dose-dependent manner. However, soluble Fn 1 marginally increased the basal level of efferocytosis in control cells (Fig. 3a). In addition, we alternatively examined the effects of Fn1 on efferocytosis by peritoneal macrophages in which Tim-4-mediated efferocytosis is well established [27]. Addition of Fn1 marginally increased efferocytosis by peritoneal macrophages derived from Tim- $4^{-1-}$ mice, but significantly increased that by peritoneal macrophages derived from wild-type (WT) mice (Fig. 3b), indicating that the effects of Fn1 on efferocytosis is Tim-4-dependent. In a reverse way, Fn1 was knocked down using $F n l$-targeting siRNA (Fig. 3c) and the effect on Tim-4-mediated efferocytosis was assessed. Knockdown of Fn1 substantially decreased Tim-4-mediated engulfment of apoptotic cells in comparison with transfection of control siRNA (Fig. 3d). Overall, the data suggest that Fn1 participates in regulation of Tim-4-mediated efferocytosis.

\section{Fn1 forms a complex with Tim-4 and integrins}

Fn1 binds to integrins involved in efferocytosis (e.g., $\alpha_{v} \beta_{5}$ and $\alpha_{v} \beta_{3}$ ), and Fn1 and integrins are reportedly involved in clearance of apoptotic cells [28-32]. Thus, we hypothesized that Fn1 brings Tim- 4 and integrins into close proximity by interaction of Fn1 with Tim- 4 and integrins, leading to effective signal transduction through integrins. In order to address this, we first 
a

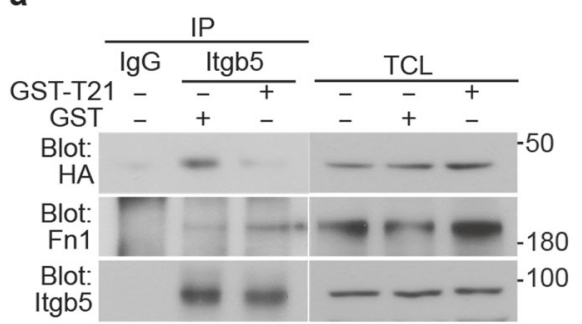

b

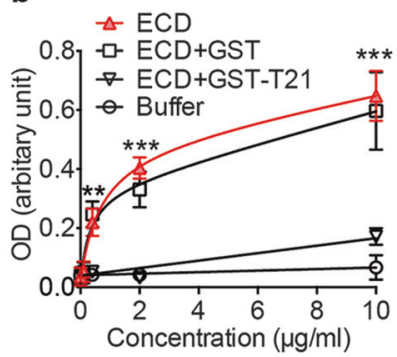

C

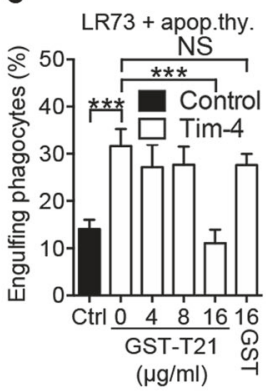

e

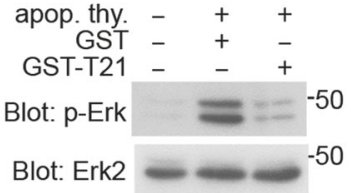

f

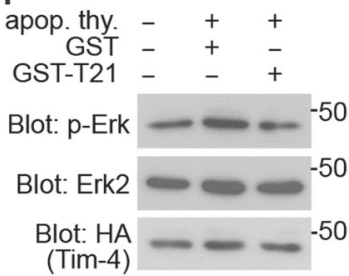

g

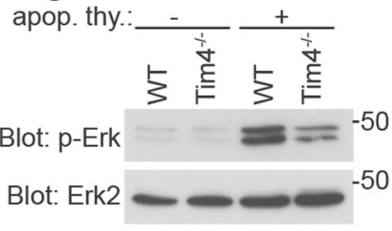

d
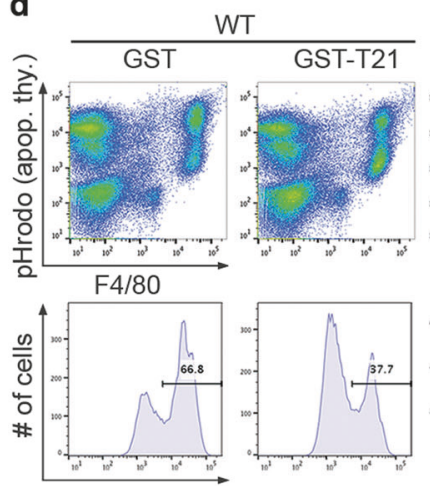

pHrodo (apop. thy.)

Fig. 4 Disruption of the interaction between Fn1 and Tim- 4 abolishes Tim-4-mediated efferocytosis and attenuates integrin signaling activation. a Lysates of LR73 cells stably expressing Tim-4 were incubated with protein A/G-conjugated agarose beads and anti-Itgb5 or $\mathrm{IgG}$ in the presence of GST-T21 or GST. Bound proteins were detected by immunoblotting. IP, immunoprecipitation; TCL, total cell lysates. b The purified ECD of Tim- 4 was incubated with Fn1 attached to a 96-well plate in the presence of GST or GST-T21, and then bound proteins were detected by measuring absorbance at $450 \mathrm{~nm}$ using a microplate reader. $n=4$. c LR73 cells transfected with Tim- 4 were incubated with apoptotic thymocytes in the presence of GST or GSTT21, and then stained with an anti-HA antibody. Engulfing phagocytes were detected by flow cytometry. $n=3$. d pHrodo-stained apoptotic thymocytes with GST or GST-T21 were injected into the peritoneum

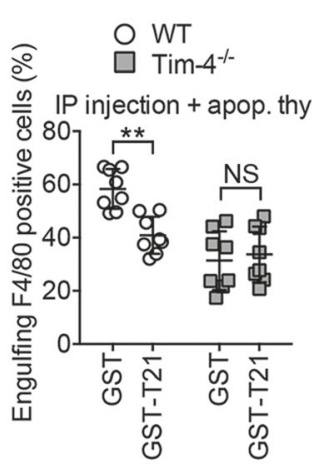

of WT or Tim- $4^{-1-}$ mice. Peritoneal exudates were prepared $15 \mathrm{~min}$ later, stained with an anti-F4/80 antibody, and analyzed by flow cytometry. Representative flow cytometric data are shown (left). $n=8$. e, f Peritoneal macrophages e or LR73 cells stably expressing Tim-4 f were incubated with apoptotic thymocytes and GST or GST-T21 for $15 \mathrm{~min}$, washed to remove unbound apoptotic cells, and then lysed. Proteins in the lysates were detected by immunoblotting. g Peritoneal macrophages derived from WT or Tim- $4^{-1-}$ mice were incubated with apoptotic thymocytes for $15 \mathrm{~min}$, washed, and lysed. Phosphorylated Erk in the lysates was detected by immunoblotting. Data are shown as the mean \pm standard deviation and images are representative of at least three independent experiments. $* * P<0.01, * * * P<0.001$. ECD, the extracellular domain of Tim-4; NS, not significant; WT, wild type

\section{Disruption of the interaction of Tim-4 with Fn1 abolishes Tim-4-mediated efferocytosis and attenuates activation of integrin signaling}

Next, if notable Tim-4-mediated efferocytosis is induced by scaffolding Tim- 4 and integrins by Fn1, the interaction of Tim-4 with Fn1 could be essential for Tim-4-mediated efferocytosis. Thus, we first tested whether the association between Tim-4 and Fn1 is necessary for Tim-4-mediated efferocytosis. To this end, we used bacterially produced GST-T21, disrupting the interaction of Tim-4 with Fn1. Intriguingly, Tim-4-mediated efferocytosis was completely abolished by GST-T21 but was marginally inhibited by GST when LR73 cells expressing Tim-4 were incubated with soluble GST-T21 or GST (Fig. 4c). To investigate the effects of disrupting this interaction in vivo, apoptotic thymocytes and GST or GST-T21 were injected into the peritoneum of 

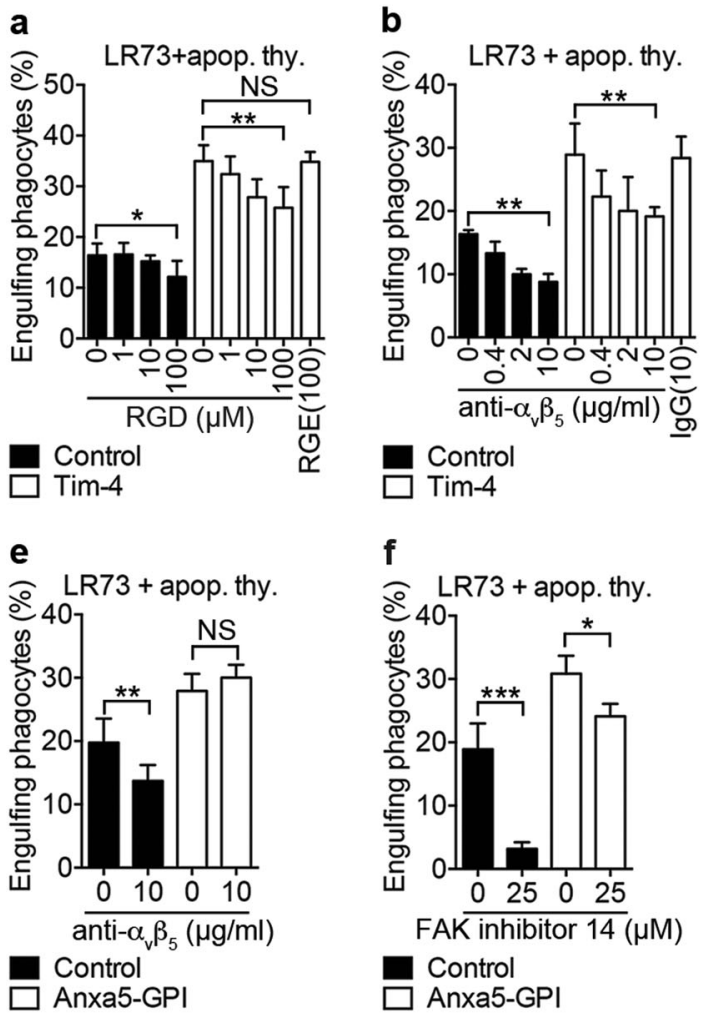

Fig. 5 Perturbation of integrin signaling decreases Tim-4-mediated efferocytosis. a-f LR73 cells transfected with Tim-4 a-c or Anxa5GPI d-f were incubated with TAMRA-labeled apoptotic thymocytes and the indicated concentrations of peptides $\mathbf{a}, \mathbf{d}$, antibodies $\mathbf{b}, \mathbf{e}$, or FAK inhibitor $14 \mathbf{c}, \mathbf{f}$ for $2 \mathrm{~h}$, stained with an anti-HA antibody, and analyzed by flow cytometry. $n=3$. g, h WT or Tim- $4^{-l-}$ mice were peritoneally injected with pHrodo-stained apoptotic thymocytes and

WT or Tim- $4^{-1-}$ mice, and efferocytosis by peritoneal macrophages was assessed. Surprisingly, GST-T21 markedly inhibited engulfment of apoptotic cells by WT peritoneal macrophages, whereas efferocytosis by $\mathrm{Tim}-4^{-1-}$ peritoneal macrophages was unaffected by GST-T21 (Fig. 4d), which suggests that the association between Fn1 and Tim-4 plays a pivotal role for Tim-4-mediated efferocytosis.

Furthermore, we investigated whether disrupting the interaction could attenuate activation of integrin signaling during efferocytosis. In order to test integrin signaling activation, we measured phosphorylation of focal adhesion kinase (FAK) and extracellular signal-regulated kinase (Erk). When peritoneal macrophages were stimulated with apoptotic cells in the presence of GST, distinct Erk and FAK phosphorylation were observed, but the levels of this phosphorylation were mitigated by adding GST-T21 (Fig. 4e and S11a). Furthermore, these phenomena were also observed in LR73 cells stably expressing Tim-4. Erk and FAK phosphorylation induced by incubation of apoptotic cells was decreased in the presence of GST-T21 (Fig. 4f and S11b). Taken together, the data suggest that the association of Tim-4 with Fn1 is necessary for Tim-4-
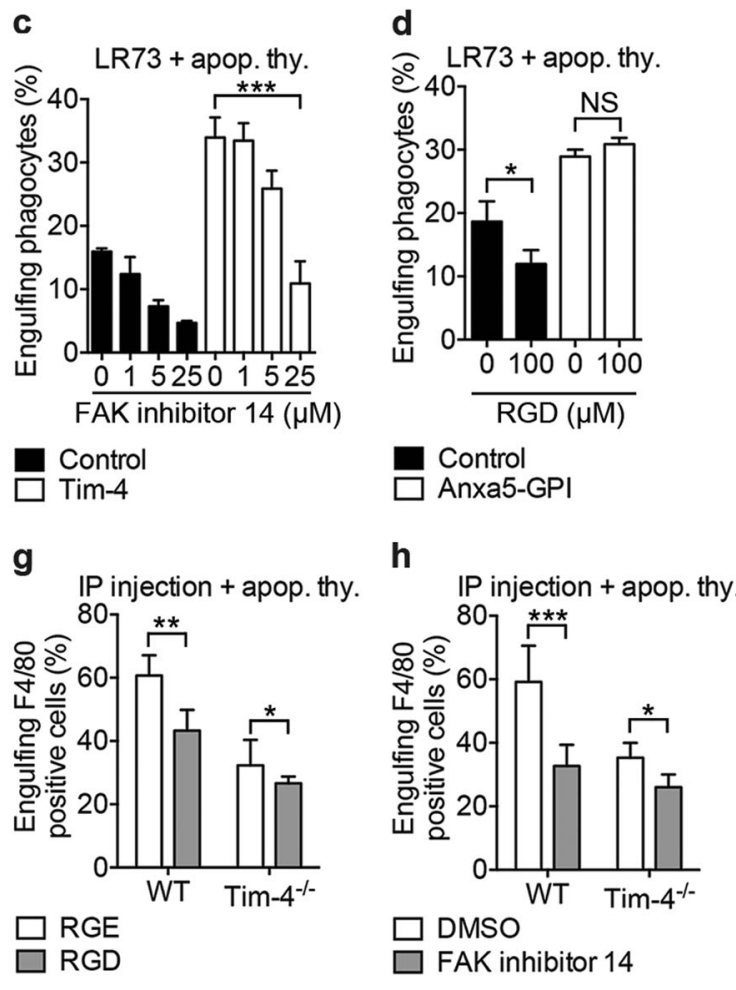

the indicated peptides $\mathbf{g}, n=7$ or FAK inhibitor $14 \mathbf{h}, n=9$. Peritoneal lavages were prepared 15 min later, stained with an anti-F4/80 antibody, and analyzed by flow cytometry. F4/80- and pHrodo-positive cells were considered to be peritoneal macrophages engulfing apoptotic cells. Data are shown as the mean \pm standard deviation. $* P<0.05$, $* * P<0.01, * * * P<0.001$. NS, not significant; WT, wild type

mediated engulfment of apoptotic cells and that Tim-4mediated efferocytosis is linked to integrin signaling through Fn1-Tim-4 association.

\section{Tim-4 depletion attenuates activation of integrin signaling}

To determine whether depletion of Tim-4 decreases integrin signaling activation, we next investigated activation of integrin signaling in $\mathrm{Tim}-4^{-1-}$ peritoneal macrophages during efferocytosis. The level of phospho-FAK was $\sim 2.5$ fold higher in WT peritoneal macrophages incubated with apoptotic cells than in those not incubated with apoptotic cells. However, incubation with apoptotic cells only increased the level of phospho-FAK by $\sim 1.5$-fold in Tim- 4 ${ }^{-1}$ peritoneal macrophages, despite there being no discernable difference in the level of basal FAK activation between WT and Tim- $4^{-1-}$ peritoneal macrophages (Fig. S12). Tim-4 dependent Erk activation during efferocytosis was more obvious than that of FAK. Upon incubation with apoptotic thymocytes, Erk phosphorylation was remarkably increased in WT peritoneal macrophages. However, Erk 
phosphorylation in Tim- $4^{-1-}$ peritoneal macrophages was not comparable with that in WT peritoneal macrophages although Erk phosphorylation in Tim- $4^{-1-}$ peritoneal macrophages was substantially increased as well (Fig. 4g). Collectively, these data demonstrate that depletion of Tim-4 attenuates activation of integrin signaling and suggest that integrin signaling activation during efferocytosis is considerably dependent on Tim-4.

\section{Blockade of integrin signaling decreases Tim-4- mediated efferocytosis}

Next, we tested whether blockade of integrin signaling using arginylglycylaspartic acid (RGD) peptides, anti- $\alpha_{\mathrm{v}} \beta_{5^{-}}$ blocking antibody, and FAK inhibitor 14 could suppress Tim-4-mediated efferocytosis. Tim-4-mediated efferocytosis was decreased by RGD peptides, but not by RGE peptides (Fig. 5a). In addition, integrin signaling was alternatively inhibited using an anti- $\alpha_{v} \beta_{5}$-blocking antibody owing to the expression of $\alpha_{\mathrm{v}} \beta_{5}$ but not $\alpha_{\mathrm{v}} \beta_{3}$ in LR73 cells (Fig. S8). Consistently, Tim-4-mediated efferocytosis was substantially decreased by treatment with an anti- $\alpha_{\mathrm{v}} \beta_{5^{-}}$ blocking antibody (Fig. 5b) but was unaffected by treatment with an anti- $\alpha_{\mathrm{v}} \beta_{3}$-blocking antibody (Fig. S13). Furthermore, FAK inhibitor 14 also abolished Tim-4-mediated engulfment of apoptotic cells (Fig. 5c). However, surprisingly, the inhibitory effects of RGD or the $\alpha_{v} \beta_{5}$-blocking antibody on Anxa5-GPI-promoted efferocytosis were not observed (Fig. 5d, e) and the effects of FAK inhibitor 14 were much less potent in Anxa5-GPI-mediated efferocytosis than in Tim-4-mediated efferocytosis (Fig. 5f), indicating the link of Tim-4-mediated efferocytosis to integrin signaling.

To investigate the effects of perturbing integrin signaling in physiologically relevant conditions, pHrodostained apoptotic thymocytes and RGD peptides or FAK inhibitor 14 were injected into the peritoneum of WT or Tim- $4^{-1-}$ mice, and efferocytosis by peritoneal macrophages was evaluated. RGD peptides and FAK inhibitor 14 strongly suppressed efferocytosis by WT peritoneal macrophages and also hindered that by Tim- $4^{-1-}$ peritoneal macrophages. However, the degree of inhibition in efferocytosis by Tim- $4^{-1-}$ peritoneal macrophages was much weaker than in that by WT peritoneal macrophages (Fig. 5g, h), which indicates that integrin signaling plays an important role even though signal transduction for Tim-4-mediated efferocytosis is not fully dependent on it. In conclusion, these data suggest that Fn1 forms a complex with Tim-4, which brings Tim-4 into close proximity with integrins. Thus, Tim-4 and integrins efficiently cooperate to secure and engulf apoptotic cells, which underlies the marked level of Tim-4-mediated efferocytosis.

\section{Discussion}

Tim-4-GPI, which comprises the ECD of Tim-4 fused to the GPI anchor of DAF, rather than full-length Tim-4 was used in this study. In this way, it was possible to determine whether the ECD of Tim-4 by itself induces a higher level of efferocytosis than Anxa5-GPI and whether it associates with a co-receptor or a protein, which further promotes Tim-4-mediated efferocytosis. Our previous study using Anxa5-GPI suggests that co-receptors physically interacting with a tethering receptor are dispensable for tethering receptor-mediated efferocytosis [19]. In fact, the previous study by our group supported two-step engulfment by Nagata group. However, a tethering receptor might still possess a co-receptor or other supplementary devices. The supplementary machinery including a scaffolding protein as in Tim-4 or a co-receptor could be beneficial for tethering receptor-mediated efferocytosis. By possessing it, tethering receptors transduce signals into phagocytes more rapidly and efficiently than two-step engulfment, resulting in notable uptake of apoptotic cells. Therefore, although Tim-4 itself is enough to promote clearance of apoptotic cells, Tim-4 could become more competent with supplementary machinery such as Fn1.

It has been suggested that integrins, such as $\alpha_{\mathrm{v}} \beta_{3}$ and $\beta_{1}$, are involved in the signaling of Tim-4-mediated efferocytosis [20, 33, 34]. Integrins were initially thought to be bystander receptors of Tim- 4 in the two-step engulfment of apoptotic cells. Specifically, it was thought that Tim-4 captures apoptotic cells, which are thereafter efficiently recognized and engulfed via integrins, and that this process does not require the direct interaction of integrins and Tim-4 [20]. Later, two groups reported that $\alpha_{\mathrm{v}} \beta_{3}$ and $\beta_{1}$ biochemically associate with Tim-4 [33, 34]. However, the direct association between Tim-4 and integrins should be carefully re-examined because there is a lack of direct biochemical data and its physiological relevance remains unclear. Although the direct biochemical interaction between Tim-4 and integrins has remained obscure, integrins are apparently involved in the signaling of Tim-4mediated efferocytosis. Our observations also support this notion. However, rather than a direct biochemical interaction, we show that Fn1 mediates the association between Tim-4 and integrins. Several reports show that Fn1 augments engulfment of apoptotic cells by associating with integrins [30-32], which is consistent with our findings.

Based on our study and others, the IgV domain of Tim-4 binds to both Fn1 and PS. Thus, it is plausible that Fn1 could compete with PS for binding to the $\operatorname{IgV}$ domain of Tim-4. However, this is not the case. Interaction of the PS binding mutant (Tim-4 ${ }^{\text {AAA }}$ ECD) with Fn1 was comparable with that of wild-type Tim-4 ECD. Moreover, LR73 cells expressing Tim-4 still promoted uptake of PS vesicles in the 
presence of Fn1 (Fig. S14), which indicates that Fn1 forms a complex with Tim- 4 without affecting the PS binding of Tim-4.

Fn1 comprises three types of domains: I, II, and III. In general, type I and II domains associate with ECM proteins such as collagen and heparin, and are involved in ECM assembly, whereas type III domains interact with various integrins via an RGD motif and thus are responsible for cell binding $[28,35]$. T21 and T48, which were identified by a yeast two-hybrid screen in this study, contain amino acids 725-889 and 841-999 of Fn1, respectively, which are located within the type III domains. A single type III domain could interact with Tim-4, and Fn1 contains multiple type III domains. Therefore, multiple molecules of Tim-4 may cluster together, resulting in the co-recruitment of different types of engulfment receptors and their synergistic coordination to tether and ingest apoptotic cells.

Collectively, the findings of this study suggest that Fn1 functions as a scaffolding protein and forms a complex with Tim- 4 and integrins. The scaffolding of these two types of engulfment receptors efficiently and synergistically coordinates the tethering and ingestion of apoptotic cells, which underlies the marked level of Tim-4-mediated efferocytosis. Given that Tim-4 is essential for engulfment of apoptotic cells, these findings could help to develop therapeutics for diseases caused by defects in efferocytosis.

\section{Materials and methods}

\section{Yeast two-hybrid screen}

The yeast two-hybrid screen was performed as described previously [36]. In brief, HF7C cells were transformed with mouse-7-embryonic library and the ECD of Tim-4 as bait using a LiAc-based method. More than 15 million independent colonies were screened and prey plasmids from yeast colonies growing on the selective plates containing 5 mM 3-amino-1,2,4-triazole without His, Trp, and Leu were recovered and amplified in $\mathrm{KC} 8$, a bacterial strain. Specific interaction was confirmed by re-transformation of yeast with the prey plasmids only or the prey plasmids with the ECD of Tim- 4 and then the inserts of the prey plasmids were identified by sequencing.

\section{Surface plasmon resonance}

Interaction between the ECD of Tim- 4 with PS or PC and interaction of Anxa5 with PS or PS were analyzed using a surface plasmon resonance instrument (SR7500 DC, Reichert Inc) according the manufacturer's instructions. In brief, PS or PC were purchased from Avanti and immobilized on planar hydrophobic sensor chip mounted on the instrument using immersion oil. Different concentrations of the purified ECD of Tim- 4 or Anxa5 in 10 mM HEPES buffer (pH 7.4) containing $140 \mathrm{mM} \mathrm{NaCl}$, $2.5 \mathrm{mM} \mathrm{CaCl}_{2}$ and $0.005 \%$ tween 20 were allowed to flow over the surface containing the immobilized lipids at a rate of $30 \mu \mathrm{l} / \mathrm{min}$ at $25^{\circ} \mathrm{C}$. The sensor surface was regenerated after each association and dissociation cycle. Sensorgrams were fit to a simple 1:1 Langmuir interaction model using data analysis program Scrubber 2.0 (BioLogic Software).

\section{Statistical analysis}

All data are shown as the mean \pm standard deviation. Each experiment was performed independently at least three times, and a two-tailed $t$ test was used to analyze statistical differences. Statistical significance was calculated using the GraphPad Prism 6 software and $p<0.05$ was taken to indicate a significant difference.

Acknowledgements This work was supported by the National Research Foundation of Korea funded by the Korea government (MSIP) (2012R1A5A1048236, 2016R1A2B4009737, and 2016M3C7A1905475) and by Aging Research Institute at GIST.

Author contributions J. L., B. P., and J. P. designed and performed research; B. M., H. M., K. K., S-A. L., D. K., and C. M. performed experiments; J. L., B. P., J. P., B. M., H. M., K. K., S-A. L., D. K., C. M., D. L., G. L, and D. P. analyzed data and discussed results; J. L, J. P., and D. P. conceived the study and wrote the manuscript.

\section{Compliance with ethical standards}

Conflict of interest The authors declare that they have no conflict of interest.

\section{References}

1. Savill J, Fadok V. Corpse clearance defines the meaning of cell death. Nature. 2000;407:784-8.

2. Hochreiter-Hufford A, Ravichandran KS. Clearing the dead: apoptotic cell sensing, recognition, engulfment, and digestion. Cold Spring Harb Perspect Biol. 2013;5:a008748.

3. Nagata S, Hanayama R, Kawane K. Autoimmunity and the clearance of dead cells. Cell. 2010;140:619-30.

4. Suzuki J, Nagata S. Phospholipid scrambling on the plasma membrane. Methods Enzymol. 2014;544:381-93.

5. Penberthy KK, Ravichandran KS. Apoptotic cell recognition receptors and scavenger receptors. Immunol Rev. 2016;269:4459.

6. Platt N, da Silva RP, Gordon S. Recognizing death: the phagocytosis of apoptotic cells. Trends Cell Biol. 1998;8:365-72.

7. Park D, Tosello-Trampont AC, Elliott MR, Lu M, Haney LB, Ma $\mathrm{Z}$, et al. BAI1 is an engulfment receptor for apoptotic cells upstream of the ELMO/Dock180/Rac module. Nature. 2007;450:430-4.

8. Miyanishi M, Tada K, Koike M, Uchiyama Y, Kitamura T, Nagata S. Identification of Tim4 as a phosphatidylserine receptor. Nature. 2007;450:435-9. 
9. Park SY, Jung MY, Kim HJ, Lee SJ, Kim SY, Lee BH, et al. Rapid cell corpse clearance by stabilin-2, a membrane phosphatidylserine receptor. Cell Death Differ. 2008;15:192-201.

10. Murakami Y, Tian L, Voss OH, Margulies DH, Krzewski K, Coligan JE. CD300b regulates the phagocytosis of apoptotic cells via phosphatidylserine recognition. Cell Death Differ. 2014;21:1746-57.

11. He M, Kubo H, Morimoto K, Fujino N, Suzuki T, Takahasi T, et al. Receptor for advanced glycation end products binds to phosphatidylserine and assists in the clearance of apoptotic cells. EMBO Rep. 2011;12:358-64.

12. Akakura S, Singh S, Spataro M, Akakura R, Kim JI, Albert ML, et al. The opsonin MFG-E8 is a ligand for the alphavbeta5 integrin and triggers DOCK180-dependent Rac1 activation for the phagocytosis of apoptotic cells. Exp Cell Res. 2004;292: 403-16.

13. Scott RS, McMahon EJ, Pop SM, Reap EA, Caricchio R, Cohen PL, et al. Phagocytosis and clearance of apoptotic cells is mediated by MER. Nature. 2001;411:207-11.

14. Ishimoto $\mathrm{Y}$, Ohashi K, Mizuno K, Nakano T. Promotion of the uptake of PS liposomes and apoptotic cells by a product of growth arrest-specific gene, gas6. J Biochem. 2000;127: 411-7.

15. Hanayama R, Tanaka M, Miwa K, Shinohara A, Iwamatsu A, Nagata S. Identification of a factor that links apoptotic cells to phagocytes. Nature. 2002;417:182-7.

16. Weinger JG, Brosnan CF, Loudig O, Goldberg MF, Macian F, Arnett HA, et al. Loss of the receptor tyrosine kinase Axl leads to enhanced inflammation in the CNS and delayed removal of myelin debris during experimental autoimmune encephalomyelitis. J Neuroinflamm. 2011;8:49.

17. Arandjelovic S, Ravichandran KS. Phagocytosis of apoptotic cells in homeostasis. Nat Immunol. 2015;16:907-17.

18. Devitt A, Moffatt OD, Raykundalia C, Capra JD, Simmons DL, Gregory CD. Human CD14 mediates recognition and phagocytosis of apoptotic cells. Nature. 1998;392:505-9.

19. Park B, Lee J, Moon H, Lee G, Lee DH, Cho JH, et al. Coreceptors are dispensable for tethering receptor-mediated phagocytosis of apoptotic cells. Cell Death Dis. 2015;6:e1772.

20. Toda S, Hanayama R, Nagata S. Two-step engulfment of apoptotic cells. Mol Cell Biol. 2012;32:118-25.

21. Nishi C, Toda S, Segawa K, Nagata S. Tim4- and MerTKmediated engulfment of apoptotic cells by mouse resident peritoneal macrophages. Mol Cell Biol. 2014;34:1512-20.

22. Yanagihashi Y, Segawa K, Maeda R, Nabeshima YI, Nagata S. Mouse macrophages show different requirements for phosphatidylserine receptor Tim4 in efferocytosis. Proc Natl Acad Sci USA. 2017;114:8800-5.
23. Kuchroo VK, Dardalhon V, Xiao S, Anderson AC. New roles for TIM family members in immune regulation. Nat Rev Immunol. 2008;8:577-80.

24. Kobayashi N, Karisola P, Pena-Cruz V, Dorfman DM, Jinushi M, Umetsu SE, et al. TIM-1 and TIM-4 glycoproteins bind phosphatidylserine and mediate uptake of apoptotic cells. Immunity. 2007;27:927-40.

25. Santiago C, Ballesteros A, Martinez-Munoz L, Mellado $\mathrm{M}$, Kaplan GG, Freeman GJ, et al. Structures of T cell immunoglobulin mucin protein 4 show a metal-Ion-dependent ligand binding site where phosphatidylserine binds. Immunity. 2007;27: 941-51.

26. Park D, Hochreiter-Hufford A, Ravichandran KS. The phosphatidylserine receptor TIM-4 does not mediate direct signaling. Curr Biol. 2009;19:346-51.

27. Wong K, Valdez PA, Tan C, Yeh S, Hongo JA, Ouyang W. Phosphatidylserine receptor Tim- 4 is essential for the maintenance of the homeostatic state of resident peritoneal macrophages. Proc Natl Acad Sci USA. 2010;107:8712-7.

28. Pankov R, Yamada KM. Fibronectin at a glance. J Cell Sci. 2002;115:3861-3.

29. Zollinger AJ, Smith ML. Fibronectin, the extracellular glue. Matrix Biol. 2017;60-61:27-37.

30. Vernon-Wilson EF, Aurade F, Brown SB. CD31 promotes beta1 integrin-dependent engulfment of apoptotic Jurkat $\mathrm{T}$ lymphocytes opsonized for phagocytosis by fibronectin. J Leukoc Biol. 2006;79:1260-7.

31. Vernon-Wilson EF, Aurade F, Tian L, Rowe IC, Shipston MJ, Savill J, et al. CD31 delays phagocyte membrane repolarization to promote efficient binding of apoptotic cells. J Leukoc Biol. 2007;82:1278-88.

32. McCutcheon JC, Hart SP, Canning M, Ross K, Humphries MJ, Dransfield I. Regulation of macrophage phagocytosis of apoptotic neutrophils by adhesion to fibronectin. J Leukoc Biol. 1998;64:600-7.

33. Flannagan RS, Canton J, Furuya W, Glogauer M, Grinstein S. The phosphatidylserine receptor TIM4 utilizes integrins as coreceptors to effect phagocytosis. Mol Biol Cell. 2014;25:1511-22.

34. Zhang Q, Wang H, Wu X, Liu B, Liu W, Wang R, et al. TIM-4 promotes the growth of non-small-cell lung cancer in a RGD motif-dependent manner. $\mathrm{Br} \mathrm{J}$ Cancer. 2015;113: 1484-92.

35. Wierzbicka-Patynowski I, Schwarzbauer JE. The ins and outs of fibronectin matrix assembly. J Cell Sci. 2003;116:3269-76.

36. Lee J, Park B, Kim G, Kim K, Pak J, Kim K, et al. Arhgef16, a novel Elmo1 binding partner, promotes clearance of apoptotic cells via RhoG-dependent Rac1 activation. Biochim Biophys Acta. 2014;1843:2438-47. 